Bull. Austral. Math. Soc.

$60 \mathrm{E} 05$

VOL. 58 (1998) [239-244]

\title{
TAIL PROBABILITIES FOR WEIGHTED SUMS OF PRODUCTS OF NORMAL RANDOM VARIABLES.
}

\author{
B. Gail Ivanoff and N.C. Weber
}

\begin{abstract}
Weighted sums of products of independent normal random variables arise naturally as distributional limits for various statistics. This note investigates the rate at which the tail probability of these sums approaches zero.
\end{abstract}

\section{INTRODUCTION}

Random variables of the form $\sum_{n=1}^{\infty} \lambda_{n} B_{n} C_{n}$, where $\left(B_{n}\right)$ and $\left(C_{n}\right)$ are independent sequences of independent, zero mean, normal random variables and $\left(\lambda_{n}\right)$ is a square summable sequence of non-negative constants occur as distributional limits for various statistics. Kallenberg [6] has shown that terms of this form arise in the characterisation of continuous, separately exchangeable processes and so these distributions can arise when studying processes that involve multiparameter indexing sets and certain forms of exchangeability. Examples of statistics of this type can be found in the study of generalised $U$-statistics $[\mathbf{1}, \mathbf{9}]$, in the study of symmetric statistics [10] and in the study of row and column exchangeable processes [4].

There has been considerable attention paid to the order of the tail probabilities of random variables of the form $\sum_{n=1}^{\infty} \lambda_{n}\left(B_{n}^{2}-E B_{n}^{2}\right)$ that arise in the study of quadratic forms (see, for example, [2]) and while the distribution of the product of two normal random variables has been investigated and tabulated (see, for example, [8]) the weighted sum of products has not.

In this paper we shall establish the exact order of the tail probabilities for the weighted sums of products of normal random variables. This result has potential applications in a number of different areas. For example, it is used in [5] to establish tightness when proving invariance principles for statistics with limiting distributions of the above form, and knowledge of the exact tail behaviour is needed when calculating asymptotic relative efficiencies for such statistics.

Received 11th February, 1998

Research for the first author supported by a grant from the Natural Sciences and Engineering Research Council of Canada. Research for the second author supported by a grant from the Australian Research Council.

Copyright Clearance Centre, Inc. Serial-fee code: 0004-9729/98 \$A2.00+0.00. 


\section{Main Result}

Let $\left(B_{n}\right)$ and $\left(C_{n}\right), n=1,2, \ldots$ be two independent sequences of independent $N(0,1)$ random variables. First we shall consider the finite sum of products $W=\sum_{j=1}^{n} B_{j} C_{j}$. Note that $W$ is symmetric about 0 . The following Lemma establishes the rate at which the tail probabilities of $W$ approach 0 .

LEMMA. For $n \geqslant 1$,

$$
\lim _{x \rightarrow \infty} x^{1-(n / 2)} e^{x} P(|W|>x)=\left[2^{(n / 2)-1} \Gamma\left(\frac{n}{2}\right)\right]^{-1} .
$$

In particular, if $n=2$ then $P(|W|>x)=e^{-x}$.

Proof: As noted in Kendall and Stuart [7, p.290], $W$ is equal in distribution to the difference $U-V$, where $U$ and $V$ are i.i.d. $\operatorname{gamma}(n / 2,1)$ random variables. Thus for $x>0, W$ has density

$$
f(x)=c e^{-x} \int_{0}^{\infty}\left(x y+y^{2}\right)^{(n / 2)-1} e^{-2 y} d y
$$

where $c=\Gamma(n / 2)^{-2}$. So

$$
f(x)=c x^{n-1} e^{-x} \int_{0}^{\infty}\left(v+v^{2}\right)^{(n / 2)-1} e^{-2 v x} d v .
$$

Thus if $n=2$ then $P(|W|>x)=e^{-x}$.

If $n=1$, then from (1)

$$
\begin{aligned}
P(|W|>x) & =\frac{2}{\pi} \int_{x}^{\infty} \int_{0}^{\infty}\left(v+v^{2}\right)^{-1 / 2} e^{-(2 v+1) u} d v d u \\
& =\frac{2}{\pi} \int_{0}^{\infty}\left(v+v^{2}\right)^{-1 / 2}(2 v+1)^{-1} e^{-(2 v+1) x} d v .
\end{aligned}
$$

Thus,

$$
\begin{aligned}
\lim _{x \rightarrow \infty} x^{1 / 2} e^{x} P(|W|>x) & =\lim _{x \rightarrow \infty} \frac{2 x^{1 / 2}}{\pi} \int_{0}^{\infty}\left(v+v^{2}\right)^{-1 / 2}(2 v+1)^{-1} e^{-2 v x} d v \\
& =\lim _{x \rightarrow \infty} \frac{\sqrt{2}}{\pi} \int_{0}^{\infty} t^{-1 / 2}\left(1+\frac{t}{2 x}\right)^{-1 / 2}\left(1+\frac{t}{x}\right)^{-1} e^{-t} d t \\
& =\sqrt{\frac{2}{\pi}}
\end{aligned}
$$

Finally, if $n>2$ then

$$
P(|W|>x)=2 c \int_{0}^{\infty}\left(v+v^{2}\right)^{(n / 2)-1}\left(\int_{x}^{\infty} u^{n-1} e^{-(2 v+1) u} d u\right) d v
$$

Using integration by parts it is easy to show that 


$$
\begin{aligned}
\lim _{x \rightarrow \infty} x^{1-(n / 2)} e^{x} P(|W|>x) & =\lim _{x \rightarrow \infty} 2 c \int_{0}^{\infty}\left(v+v^{2}\right)^{(n / 2)-1} x^{n / 2}(2 v+1)^{-1} e^{-2 v x} d v \\
& =2^{1-(n / 2)} / \Gamma\left(\frac{n}{2}\right)
\end{aligned}
$$

Let $\left(\lambda_{n}\right), n=1,2,3, \ldots$ be a nonincreasing sequence of positive real numbers. Let $\xi_{1}=\lambda_{1}=\ldots=\lambda_{n_{1}}>\lambda_{n_{1}+1}$. Similarly, we define $\xi_{j}$ to be the $j^{\text {th }}$ largest of the $\lambda_{n}$ and $n_{j}$ its multiplicity. Let $S=\sum_{i=1}^{\infty} \lambda_{i} B_{i} C_{i}$. Thus, $S=\sum_{j=1}^{\infty} \xi_{j} W_{j}$ where $\xi_{1}>\xi_{2}>\ldots \geqslant 0$ and $W_{j}=\sum_{i=n_{1}+\ldots+n_{j-1}+1}^{n_{1}+\ldots+n_{j}} B_{i} C_{i}$

THEOREM. Let $\left(\lambda_{n}\right), n=1,2,3, \ldots$ be a nonincreasing sequence of positive real numbers such that $\sum_{i=1}^{\infty} \lambda_{i}^{2}<\infty$. If $S=\sum_{i=1}^{\infty} \lambda_{i} B_{i} C_{i}$ then $P(|S|<\infty)=1$ and

$$
P(|S|>x)<K P\left(\left|W_{1}\right|>x / \lambda_{1}\right),
$$

where $K$ is a finite, positive constant.

Proof: That $P(|S|<\infty)=1$ is shown in [6]. It is easily shown that $S$ has moment generating function $\prod_{i=1}^{\infty}\left(1-t^{2} \xi_{i}^{2}\right)^{-n_{i} / 2}$ for $t<\xi_{1}^{-1}$.

We observe first that $S$ has a density $f_{S}$ and we shall use the method exploited by Hoeffding [3] to show that

$$
\frac{f_{S}(x)}{f(x)}=\int_{-\infty}^{\infty} \frac{f(x-z)}{f(x)} d G(z) \leqslant K, \forall x \neq 0,
$$

for some finite constant $K$, where $f$ is the density of $\xi_{1} W_{1}$ and $G$ is the distribution function of $\sum_{j=2}^{\infty} \xi_{j} W_{j}$. Thus, for $x>0$,

$$
P(|S|>x) \leqslant K P\left(\left|\xi_{1} W_{1}\right|>x\right) .
$$

In what follows, the $K_{i}$ 's are finite, positive constants although their values are not always explicitly stated.

To prove Equation (2), we again use the observation in [7] that $\xi_{1} W_{1}$ is equal in distribution to the difference of two independent gamma $\left(n_{1} / 2,1 / \lambda_{1}\right)$ random variables. Let $\kappa=\lambda_{1}^{-n_{1}} \Gamma\left(n_{1} / 2\right)^{-2}$. Then

$$
f(x)=\kappa e^{-x / \lambda_{1}} \int_{-x \vee 0}^{\infty}\left(y^{2}+x y\right)^{\left(n_{1} / 2\right)-1} e^{-2 y / \lambda_{1}} d y
$$

and so

$$
\frac{f(x-z)}{f(x)}=e^{z / \lambda_{1}} \cdot \frac{\int_{(z-x) \vee 0}^{\infty}\left(y^{2}+x y-z y\right)^{\left(n_{1} / 2\right)-1} e^{-2 y / \lambda_{1}} d y}{\int_{0}^{\infty}\left(y^{2}+x y\right)^{\left(n_{1} / 2\right)-1} e^{-2 y / \lambda_{1}} d y}
$$


since we may assume (by symmetry) that $x>0$. Thus when $n_{1}=2$

$$
\int_{0}^{\infty} \frac{f(x-z)}{f(x)} d G(z) \leqslant \int_{-\infty}^{\infty} e^{z / \lambda_{1}} d G(z)=\prod_{i=2}^{\infty}\left(1-\frac{\xi_{i}^{2}}{\xi_{1}^{2}}\right)^{-n_{i} / 2}=\zeta .
$$

That $\zeta$ is finite follows from the fact that $\xi_{1}>\xi_{i}, \forall i \geqslant 2$ and that $\sum_{i=2}^{\infty} n_{i} \xi_{i}^{2}=\sum_{i=n_{1}+1}^{\infty} \lambda_{i}^{2}<$ $\infty$.

We next prove Equation (2) when $n_{1}>2$. Consider the right hand side of Equation (4) and assume first that $z<0$.

$$
\begin{aligned}
\int_{0}^{\infty} & \left(y^{2}+x y-z y\right)^{\left(n_{1} / 2\right)-1} e^{-2 y / \lambda_{1}} d y \\
& \leqslant(1+x+|z|)^{\left(n_{1} / 2\right)-1}\left[\int_{0}^{1} y^{\left(n_{1} / 2\right)-1} e^{-2 y / \lambda_{1}} d y+\int_{1}^{\infty} y^{n_{1}-2} e^{-2 y / \lambda_{1}} d y\right] \\
& =K_{1}(1+x+|z|)^{\left(n_{1} / 2\right)-1} .
\end{aligned}
$$

On the other hand,

$$
\begin{aligned}
\int_{0}^{\infty} & \left(y^{2}+x y\right)^{\left(n_{1} / 2\right)-1} e^{-2 y / \lambda_{1}} d y \\
& \geqslant(1+x)^{\left(n_{1} / 2\right)-1}\left[\int_{0}^{1} y^{n_{1}-2} e^{-2 y / \lambda_{1}} d y+\int_{1}^{\infty} y^{\left(n_{1} / 2\right)-1} e^{-2 y / \lambda_{1}} d y\right] \\
& =K_{2}(1+x)^{\left(n_{1} / 2\right)-1} .
\end{aligned}
$$

Therefore,

$$
\int_{-\infty}^{0} \frac{f(x-z)}{f(x)} d G(z) \leqslant \int_{-\infty}^{0} \frac{K_{1}}{K_{2}}(1+|z|)^{\left(n_{1} / 2\right)-1} e^{z / \lambda_{1}} d G(z)<K_{3} .
$$

The convergence of the integral in (8) is clear since $G$ is a distribution function and $(1+|z|)^{\left(n_{1} / 2\right)-1} e^{z / \lambda_{1}}$ is bounded for $z<0$.

Now assume that $z \geqslant 0$. Since for all $y \geqslant 0,(x-z) y \leqslant x y$, we have that $f(x-z) / f(x) \leqslant e^{z / \lambda_{1}}$, and so

$$
\int_{0}^{\infty} \frac{f(x-z)}{f(x)} d G(z) \leqslant \int_{-\infty}^{\infty} e^{z / \lambda_{1}} d G(z)=\prod_{i=2}^{\infty}\left(1-\frac{\xi_{i}^{2}}{\xi_{1}^{2}}\right)^{-n_{i} / 2}=\zeta .
$$

Thus we see that for $n_{1} \geqslant 2,(2)$ is satisfied with $K=K_{3}+\zeta$.

The case $n_{1}=1$ is somewhat more delicate since in this case, $f(0)$ is undefined. Let $g$ denote the density of $\sum_{j=2}^{\infty} \xi_{j} W_{j}$. For $x>0$,

$$
\begin{aligned}
\frac{f_{S}(x)}{f(x)}= & \frac{\int_{-\infty}^{0} \int_{0}^{\infty} e^{z / \lambda_{1}}\left(y^{2}+x y-z y\right)^{-1 / 2} e^{-2 y / \lambda_{1}} g(z) d y d z}{\int_{0}^{\infty}\left(y^{2}+x y\right)^{-1 / 2} e^{-2 y / \lambda_{1}} d y} \\
& +\frac{\int_{0}^{x} \int_{0}^{\infty} e^{z / \lambda_{1}}\left(y^{2}+x y-z y\right)^{-1 / 2} e^{-2 y / \lambda_{1}} g(z) d y d z}{\int_{0}^{\infty}\left(y^{2}+x y\right)^{-1 / 2} e^{-2 y / \lambda_{1}} d y} \\
& +\frac{\int_{x}^{\infty} \int_{z-x}^{\infty} e^{z / \lambda_{1}}\left(y^{2}+x y-z y\right)^{-1 / 2} \cdot e^{-2 y / \lambda_{1}} g(z) d y d z}{\int_{0}^{\infty}\left(y^{2}+x y\right)^{-1 / 2} e^{-2 y / \lambda_{1}} d y} .
\end{aligned}
$$


The right hand side of (10) is bounded above by

$$
\frac{\int_{-\infty}^{0} e^{z / \lambda_{1}} \int_{0}^{\infty}\left(y^{2}+x y\right)^{-1 / 2} e^{-2 y / \lambda_{1}} d y g(z) d z}{\int_{0}^{\infty}\left(y^{2}+x y\right)^{-1 / 2} e^{-2 y / \lambda_{1}} d y} \leqslant \zeta .
$$

To bound (11) and (12) we shall apply Equation (5) to the sum

$$
S^{\prime}=\lambda B_{2} C_{2}+\lambda B_{3} C_{3}+\sum_{i=4}^{\infty} \lambda_{i} B_{i} C_{i}
$$

where $\lambda_{1}>\lambda>\lambda_{2}$, so that $f_{S^{\prime}}(z) \leqslant \zeta^{\prime}(2 \lambda)^{-1} e^{-|z| / \lambda}$, where $\zeta^{\prime}=\prod_{i=4}^{\infty}\left(1-\left(\xi_{i}^{2} / \lambda^{2}\right)\right)^{-n_{i} / 2}$. However, since $\lambda>\lambda_{2} \geqslant \lambda_{3}$, the density of $\lambda_{i} B_{i} C_{i}$ is uniformly bounded above by the density of $\lambda B_{i} C_{i}$ multiplied by $\lambda / \lambda_{i}, i=2,3$. Thus, there exist finite constants $K_{4}, K_{5}$ such that $g(z) \leqslant K_{4} f_{S^{\prime}}(z) \leqslant K_{5} e^{-|z| / \lambda}$. We observe also that

$$
\left[\int_{0}^{\infty}\left(y^{2}+x y\right)^{-1 / 2} e^{-2 y / \lambda_{1}} d y\right]^{-1} \leqslant K_{6} \max (1, \sqrt{x})
$$

for some constant $K_{6}$. Thus, defining $\alpha=(1 / \lambda)-\left(1 / \lambda_{1}\right)>0$ and $K_{7}=K_{5} K_{6}$,

$$
\begin{aligned}
(11) & \leqslant K_{7} \max (1, \sqrt{x}) \int_{0}^{x} \int_{0}^{\infty} e^{-\alpha z}\left(y^{2}+x y-z y\right)^{-1 / 2} e^{-2 y / \lambda_{1}} d y d z \\
& \leqslant K_{7} \max (1, \sqrt{x}) e^{-\alpha x} \int_{0}^{x} e^{\alpha(x-z)}(x-z)^{-1 / 2} d z \int_{0}^{\infty} y^{-1 / 2} e^{-2 y / \lambda_{1}} d y \\
& \leqslant K_{8} \max (1, \sqrt{x}) e^{-\alpha x} \int_{0}^{x} e^{\alpha u} u^{-1 / 2} d u \\
& \leqslant \begin{cases}K_{8} \int_{0}^{1} u^{-1 / 2} d u & \text { if } x \leqslant 1 \\
K_{8} \sqrt{x} e^{-\alpha x}\left[e^{(\alpha x / 2)} \int_{0}^{x / 2} u^{-1 / 2} d u+\sqrt{2 / x} \int_{x / 2}^{x} e^{\alpha u} d u\right] & \text { if } x>1 \\
& \leqslant K_{9} .\end{cases}
\end{aligned}
$$

Finally, making the change of variable $u=y-(z-x)$,

$$
\begin{aligned}
(12) & \leqslant K_{7} \max (1, \sqrt{x}) \int_{x}^{\infty} \int_{0}^{\infty} e^{-\alpha z}\left(u^{2}+(z-x) u\right)^{-1 / 2} e^{-2 u / \lambda_{1}} e^{-2(z-x) / \lambda_{1}} d u d z \\
& \leqslant K_{7} \max (1, \sqrt{x}) e^{-\alpha x} \int_{x}^{\infty} e^{-(z-x)\left(1 / \lambda+1 / \lambda_{1}\right)}(z-x)^{-1 / 2} d z \int_{0}^{\infty} u^{-1 / 2} e^{-2 u / \lambda_{1}} d u \\
& \leqslant K_{10} \max (1, \sqrt{x}) e^{-\alpha x} \\
(15) & \leqslant K_{11} .
\end{aligned}
$$

This completes the proof.

COROLlary 1. The tail probability $P(|S|>x)=O\left(x^{\left(n_{1} / 2\right)-1} e^{-x / \lambda_{1}}\right)$.

COROLLARY 2. Using the notation of the Theorem, if $m$ is a positive constant then

$$
E|S|^{m} \leqslant K n_{1}^{m+1} \lambda_{1}^{m} E\left|B_{1}\right|^{2 m} .
$$


PROOF: The result follows from

$$
E|S|^{m}=m \int_{0}^{\infty} x^{m-1} P(|S|>x) d x
$$

and the inequality

$$
P\left(\left|W_{1}\right|>x\right) \leqslant n_{1} P\left(\left|B_{1} C_{1}\right|>x / n_{1}\right) .
$$

\section{REFERENCES}

[1] G.K. Eagleson, 'Orthogonal expansions and U-statistics', Aust. J. Statist. 21 (1979), 221-237.

[2] G.G. Gregory, 'On efficiency and optimality of quadratic tests', Ann. Statist. 8 (1980), 116-131.

[3] W. Hoeffding, 'On a theorem of V.M. Zolotarev', Theory Probab. Appl. 9 (1964), 89-91.

[4] B.G. Ivanoff and N.C. Weber, 'Functional limit theorems for row and column exchangeable arrays', J. Multivariate Anal. 55 (1995), 133-148.

[5] B.G. Ivanoff and N.C. Weber, 'A maximal inequality and tightness for multiparameter stochastic processes', in Asymptotic methods in probability and statistics, (B. Szyszkowicz, Editor) (Elsevier Science, Amsterdam, 1998) (to appear).

[6] O. Kallenberg, 'Some new representations in bivariate exchangeability', Probab. Theory Related Fields 77 (1988), 415-455.

[7] M.G. Kendall and A. Stuart, The advanced theory of statistics, (4th edition) (Macmillan Publishing, New York, 1977).

[8] W.Q. Meeker, L.W. Cornwell and L.A. Aroian, 'The product of two normally distributed random variables', in Selected tables in mathematical statistics VII (Amer. Math. Soc., Providence, R.I., 1980).

[9] G. Neuhaus, 'Functional limit theorems for $U$-statistics in the degenerate case', J. Multivariate Anal. 7 (1977), 424-439.

[10] H. Rubin and R.A. Vitale, 'Asymptotic distribution of symmetric statistics', Ann. Statist. 8 (1980), 165-170.

Department of Mathematics and Statistics

University of Ottawa

PO Box 450 Station A

Ottawa, Ontario

Canada K1N 6N5

email: BGISG@sciences.uottawa.ca
School of Mathematics and Statistics

University of Sydney

New South Wales 2006

Australia

e-mail: neville@maths.usyd.edu.au 